\title{
PRPF31 reduction causes mis-splicing of the phototransduction genes in human organotypic retinal culture
}

\author{
Leila Azizzadeh Pormehr ${ }^{1} \cdot$ Shahin Ahmadian $\mathbb{1}^{1} \cdot$ Narsis Daftarian ${ }^{2,3} \cdot$ Seyed Ahmad Mousavi ${ }^{4}$. \\ Mahshid Shafiezadeh ${ }^{1}$
}

Received: 6 February 2019 / Revised: 30 September 2019 / Accepted: 13 October 2019 / Published online: 25 October 2019

(c) The Author(s), under exclusive licence to European Society of Human Genetics 2019

\begin{abstract}
PRPF31 is ubiquitously expressed splicing factor and has an essential role in the pre-mRNA splicing in all tissues. However, it is not clear how reduced expression of this general splicing factor leads to retinal restricted disease, retinitis pigmentosa (RP). In this study, we used RNA interference and RNA-sequencing to mimic the PRPF31 haploinsufficiency in human organotypic retinal cultures (HORCs). We examined the effects of PRPF31 deficiency on splicing by analyzing the differential exon usages (DEUs) and intron retentions of the retinal transcriptome. Our results revealed that the PRPF31 deficiency causes mis-splicing of genes involved in RNA processing (PRPF3, PRPF8, PRPF4, and PRPF19) and phototransduction (RHO, ROM1, FSCN2, GNAT2, and GNAT1) in the retina in the PRPF31 reduced samples. Mis-splicing of genes implicated in phototransduction was associated with photoreceptor degeneration observed in RP patients. Our data revealed that PRPF31 deficiency leads to the mis-splicing of a distinct subset of pre-mRNAs with a widespread effect on phototransduction and RNA processing.
\end{abstract}

\section{Introduction}

Retinitis pigmentosa (RP) is a highly heterogeneous group of retinal disorder, characterized by progressive degeneration of rod photoreceptor cells with night blindness and then with disease progression, cone photoreceptors become involved,

Supplementary information The online version of this article (https:// doi.org/10.1038/s41431-019-0531-1) contains supplementary material, which is available to authorized users.

Shahin Ahmadian

sh.ahmadian@ut.ac.ir

$\triangle$ Narsis Daftarian

nardaftarian@gmail.com

1 Department of Biochemistry, Institute of Biochemistry and Biophysics (IBB), University of Tehran, Tehran, Iran

2 Ocular Tissue Engineering Research Center, Shahid Beheshti University of Medical Sciences, Tehran, Iran

3 Ophthalmic Research Center, Shahid Beheshti University of Medical Sciences, Tehran, Iran

4 Department of Stem Cells and Developmental Biology, Cell Science Research Center, Royan Institute for Stem Cell Biology and Technology, ACECR, Tehran, Iran and eventually lead to blindness [1]. To date, variants in more than 70 genes are associated with RP (https://sph.uth.edu/ retnet) [2]. Variants in PRPFs (pre-mRNA processing factors) encoding genes represent the second most common cause for autosomal dominant RP after variants in rhodopsin [3]. These genes include PRPF3, PRPF8, PRPF4, PRPF31, PAP, and SNRN2000, encoding for the core component of the U4/U6. U5 tri-small nuclear ribonucleoproteins (snRNPs), that catalyze pre-mRNA splicing $[4,5]$.

Pre-mRNA splicing generates diversity within eukaryotic genes by differential exon usages (DEUs) and intron retentions that increases the transcript variations of the genome [6]. The retina is one of the fast metabolizing tissues in the body that has a high request for adequate expression and proper splicing of transcripts and uses its organo-specific genes. Any dysregulation of alternative splicing has been reported in RP patients [7, 8]. Furthermore, the correct regulation of splicing for photoreceptor-specific transcripts is essential for retinal maintenance and development.

Disease causing variants in PRPFs disrupt the splicesome assembly and function, leading to defect splicing of retinal specific genes or toxic function of proteins that initiate photoreceptors apoptosis $[4,9,10]$. PRPFs splicing factors are ubiquitously expressed in every cell type in the body, but it is unknown how the deficiency of these general 
splicing factors can lead to the retinal specific disease $[11,12]$. PRPFs animal models have failed to provide proper answers for the reason of the disease [13-15] and retinal biopsies to assay photoreceptors directly are not an ethically correct.

PRPF31 is a pivotal protein for the interaction between the U4/U6 disnRNP and U5 snRNP and requires for pre-mRNA splicing [16]. Reduction of the PRPF31 protein level was identified in lymphoblastoid cells of RP patients indicating that haploinsufficiency is the possible cause for RP11 [17-20].

Previously, we have reported the successful usage of the human organotypic retinal cultures (HORCs) as a model for RP11 disease [21]. In the present study, we applied RNAsequencing in the HORCs model to identify genes and molecular pathways that are altered (via differential exon usage and intron retention) in control and PRPF31 reduced samples. Transcriptome analysis showed that the reduction of PRPF31 caused mis-splicing of many genes, enriched in phototransduction and splicing program indicating the group of genes mediating the tissue-specific phenotype.

\section{Materials and methods}

\section{Human organotypic retinal culture}

Donor human eyes were obtained within the $24 \mathrm{~h}$ postmortem (20-40 years old) from Iran Eye Bank (www.ira neyebank.org) in compliance with the Declaration of Helsinki. In this study, we used the retinal tissues from donors who recorded their consent to donate their organs and tissues in the Iranian society of organ donation. The retina was isolated from the other parts of the globes, dissected and placed into serum-free Dulbecco's Modified Eagle Medium (Gibco, Thermo Fisher Scientific, Waltham, MA), RGCside up and incubated at $37^{\circ} \mathrm{C}$ in a humidified atmosphere of $95 \% \mathrm{O}_{2} / 5 \% \mathrm{CO}_{2}$.

\section{RNA interference using siRNA}

Pool siRNAs for PRPF31 (SC-62892, Lot\#L2807, Santa Cruz, CA) and pool scramble siRNAs were reverse transfected to reduce PRPF31 level in HORCs. Transfection was performed based on the calcium phosphate protocol [22] with some modifications [21]. The final concentration of siRNA was $50 \mathrm{nM}$ for each transfection.

\section{Western blot analysis}

For western blot analysis, control and treated explants were collected after $63 \mathrm{~h}$ of transfection and proteins were extracted using RIPA lysis buffer with a protease inhibitor cocktail. The concentration of total protein was measured by Bradford assay using Bovine serum albumin as a protein standard and $50 \mu \mathrm{g}$ of each sample was analyzed by western blot. All reactions were performed in biological triplicates.

\section{RNA-sequencing and quality control}

The transcriptome of control siRNA and PRPF31-reduced HORCs were sequenced on an Illumina HiSeq4000 platform, using $150 \mathrm{bp}$ paired-end reads. We performed quality control of raw Fastq files with FastQC software, and Trimmomatic V 0.36 was used to trim adapter sequences and eliminate all low-quality reads by the Phred score cutoff 20. The clean reads were aligned to human genome references 38 (hg38), using HISAT2 [23] with default parameters for finding DEUs.

\section{Identification of DEUs (differentially exon usages)}

To identify genes with DEUs between the treated and control samples, we used DEXSeq [24]. To run DEU analysis, we first quantified exon expression by performing HTSeq-count tool on HISAT2 bam files against all human exon annotation that were extracted from hg38 GTF file (downloaded from Ensemble). In the next step, DEXSeq was run in the modified version of DESeq2 package to find the differentially expressed exons. Finally, extraction of the exons, having adjusted $P$-value $<0.01$ and fold change $>2$, was carried out for further investigations. For visualization of RNA-sequencing results, we used the $\mathrm{R}$ program, pheatmap package, and ggplot 2 package. We estimated transcript assembly with Cufflinks2 [25].

\section{Analysis of differentially spliced genes}

We used HISAT2 splice junction to analysis differentially spliced genes between control and treated samples.

\section{Gene ontology analysis}

The PANTHER website was used to generate gene ontology (GO) annotation of DEUs. For GO analysis, all altered exon usages and spliced genes were selected and used in PANTHER website search engine. In order to find significant GO terms, we just relied on the terms, enriched with adjusted $P$-value $<0.01$. PANTHER adjustment method of Benjamini-Hochberg was employed for this purpose.

\section{Biological replication of differential alternative splicing results via RT-PCR}

Total RNA was extracted from control and treated samples using RNA extraction kit (TAKARA, Japan) according to the manufacturer's instructions. The RNA concentration was 
measured using a NanoDrop ND-2000 spectrophotometer (NanoDrop Technologies, USA). Total RNA (1 $\mu \mathrm{g})$ was reverse transcribed using oligo dT to cDNA. Primers were designed from the exon-intron boundary region using Primer3 (http://bioinfo.ut.ee/primer3-0.4.0/). For confirmation of target-specificity of primers, Primer-BLAST was used (http://www.ncbi.nlm.nih.gov/tools/primer-blast/). Primers are listed in Supplementary Table 1. Intron retentions were analyzed by RT-PCR with $1 \mu \mathrm{g}$ of the total RNA sample using the cDNA reverse transcription kit (Applied Biosystems) and Taq DNA polymerase (Bioflux) and gene-specific primers. A different temperatures $68-55^{\circ} \mathrm{C}$ were used to amplify the different transcripts. The PCR products were separated by electrophoresis on $2 \%$ agarose gels, using safe stein nucleic acid stein (Cinnagen) and imaging on a UV transilluminator to visualize the bands. Distinct DNA bands were purified using the Gel Extraction Kit (Cinnagen) and confirmed by Sanger sequencing.

\section{Statistical analysis}

Differences between treated and control samples were analyzed by students' $t$ test. All results were given as the mean \pm standard deviation of three $(n=3)$ independent samples (in triplicate). Differences with $P<0.01$ were considered statistically significant.

\section{Results}

\section{PRPF31 reduction in HORCs}

To analyze protein reduction in HORCs, after $63 \mathrm{~h}$ of transfection, western blot analysis was performed on the proteins, extracted from control and treated samples and the western blot analysis with ImageJ showed $60 \%$ reduction in the expression of PRPF31 protein in treated samples (Fig. 1).

\section{Transcriptome analysis of PRPF31 reduced HORC by RNA-sequencing}

RNA-sequencing was used to examine the genome-wide effect of PRPF31 reduction on exon usage in HORCs. Among ten samples for sequencing, only four samples (two control and two treated samples) had a RNA integrity number above 7 for analysis. therefore, we generated RNAsequencing from four control and PRPF31 reduced samples, using standard mRNA library preparation protocol [26]. The libraries were sequenced using an Illumina Hiseq 4000 machine (Novogene Ltd., China). Eventually, we generated $\sim 280$ million paired-end raw sequence reads of $150 \mathrm{bp}$ in length. After deleting low quality reads with Trimmomatic, 50.8 and 52.7 million reads for control

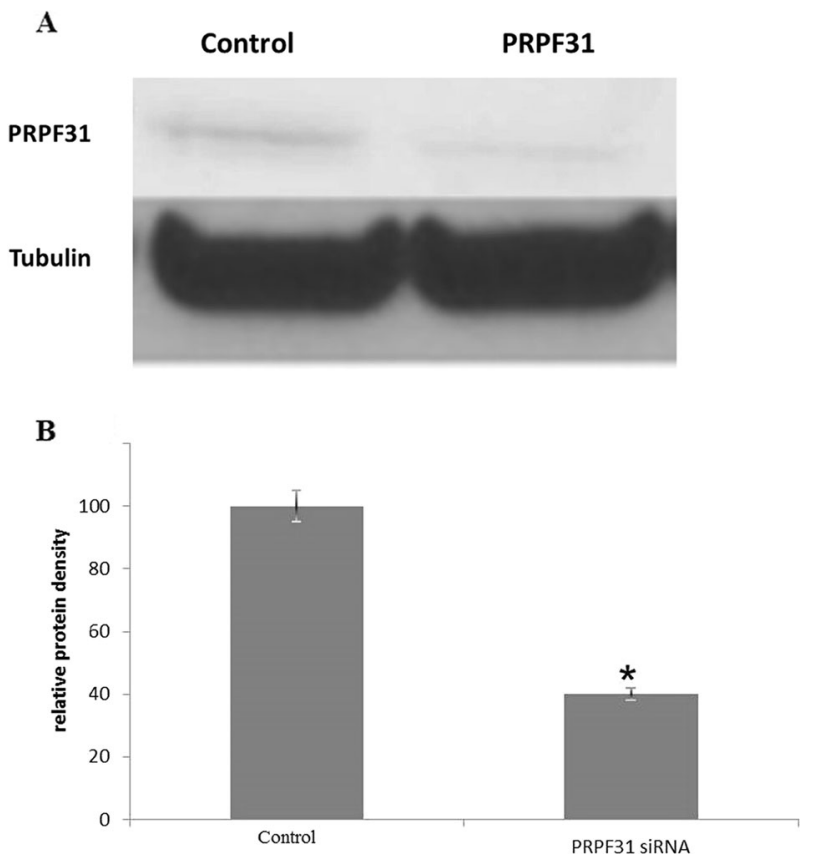

Fig. 1 PRPF31 protein is reduced after transfection a western blot analysis of PRPF31 reduction, Tubulin loaded as a control, b plot shows densitometry analysis of the corresponding protein blots by ImageJ, three independent experiments $(n=3)$ were done in triplicate, $* P<0.01$

samples and 62.8 and 101.5 million reads remained for two replicate analyses, respectively (Table 1). All clean reads had the lengths of $\sim 130-135$ bp with Phred score $>20$. The mapping rates were $>93 \%$ of the clean reads mapped to the reference genome and $\sim 82-91 \%$ of reads showed a complete match with the reference genome.

\section{Differential exon usage of genes encoding mRNA processing and phototransduction}

To study PRPF31 deficiency on alternative splicing in control and PRPF31 reduced samples, DEXSeq pipeline was performed on the RNA transcriptome data. We observed DEUs in at least one exon of the genes, involved in pre-mRNA processing factors (PRPF4, PRPF3, $P R P F 8$, and PRPF19) (Fig. 2a) and phototransduction genes (RHO, GNAT1, ROM1, FSCN2, PDE6A, and PDE6B), as compared with control in our study (Fig. 2b). We selected DEUs with fold change $\geq 2$ and $\leq 2$ and adjusted $p$-value 0.01 to find most significant DEUs. In this study, from 683,196 analyzed exons, 3170 exons showed DEU (Supplementary Table 2).

\section{Gene ontology and pathway analysis of DEUs}

We performed Gene set enrichment analysis to identify pathways and processes showing top DEUs. The results show that the PRPF31 reduction was associated with 
Table 1 Summary of RNA-seq results of reads

\begin{tabular}{llll}
\hline Sample ID & Sequencing platform & Read length (bp) & Sequencing depth (Reads) \\
\hline Control-1 & Illumina Hiseq 4000 & $150 \mathrm{bp}$ & 50855840 \\
Treat-1 & Illumina Hiseq 4000 & $150 \mathrm{bp}$ & 101536788 \\
Control-2 & Illumina Hiseq 4000 & $150 \mathrm{bp}$ & 52752393 \\
Treat-2 & Illumina Hiseq 4000 & $150 \mathrm{bp}$ & 62851720 \\
\hline
\end{tabular}

$\mathbf{A}$
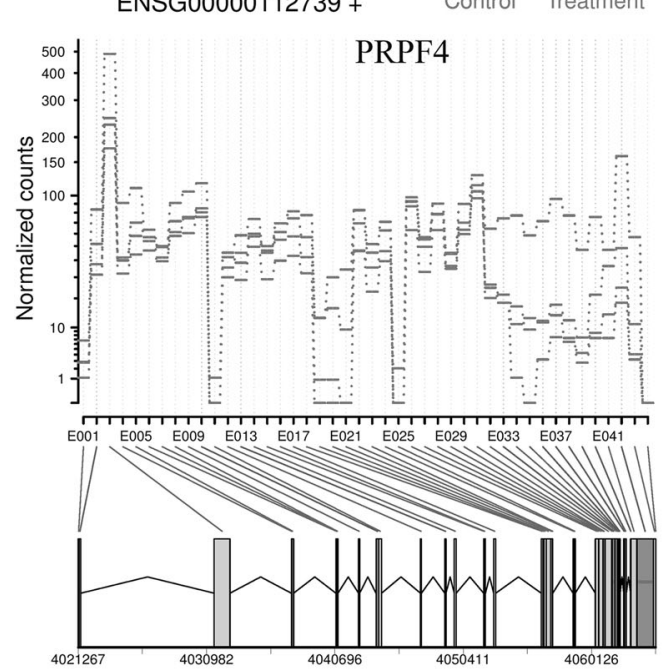

ENSG00000117360 + C Control Treatment
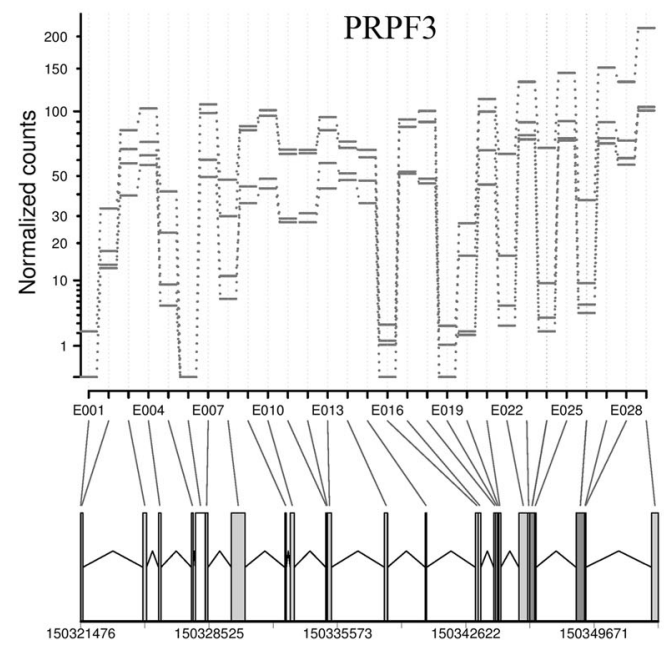

Fig. 2 DEU in genes, involved in phototransduction, and pre-mRNA splicing between control and treated samples. a DEXseq analysis on RNA-sequencing data displayed that $P R P F 31$ reduction significantly altered exon usage of $P R P F 3, P R P F 8, P R P F 4$, and $P R P F 19$ involved

deregulation of many genes and pathways, including another RNA processing factors such as PRPF4, PRPF3, PRPF8, PRPF 19, photoreceptor cell development, phototransduction, and proteasome-mediated ubiquitin-dependent protein catabolic process (Table 2).
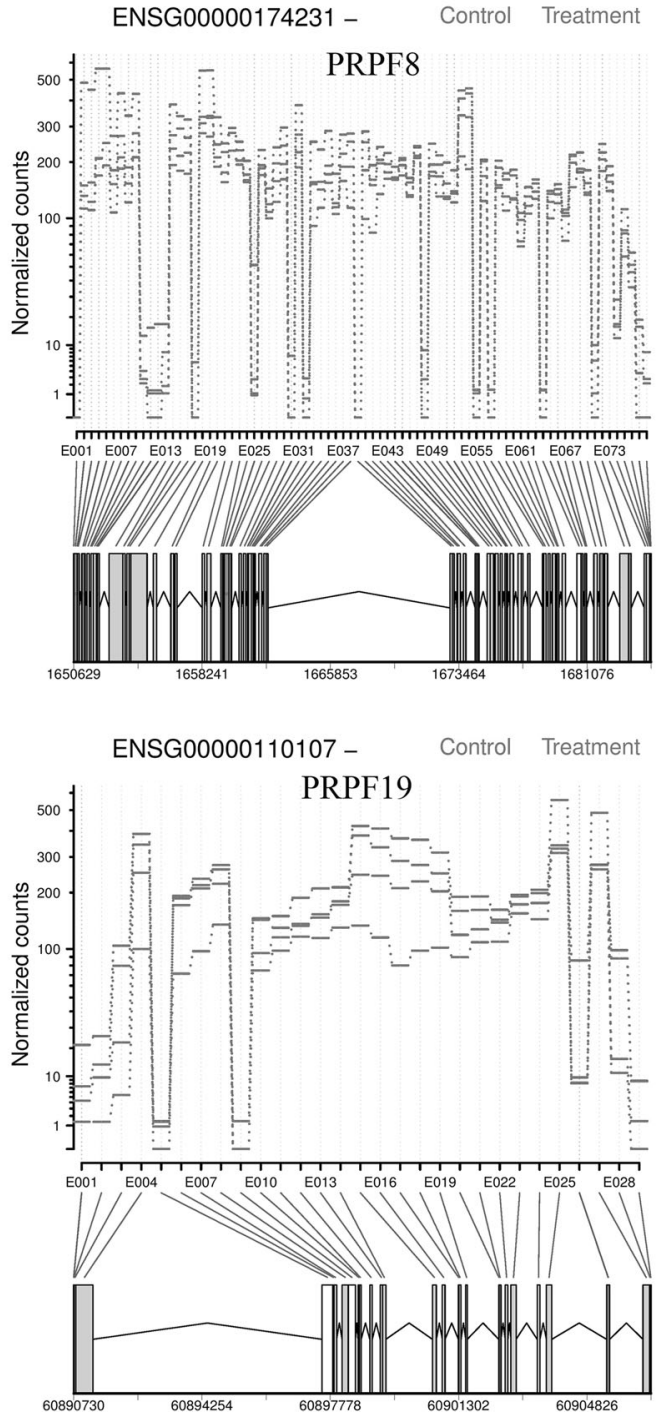

in pre-mRNA splicing. b DEU in RHO, FSCN2, ROM1, GNAT1, $P D E 6 A$, and $P D E 6 B$ genes involved in phototransduction, $63 \mathrm{~h}$ post transfection. The exons, highlighted in purple, represent the significant differential exon usage

Using PANTHER functional annotation tool, we observed significant enrichment of cellular component including axonal growth cone, photoreceptor cell cilium, cytoplasmic ribonucleoprotein granule, and mitochondrial outer membrane (Supplementary Table 3). 
B ENSG00000186765+ Control Treatment
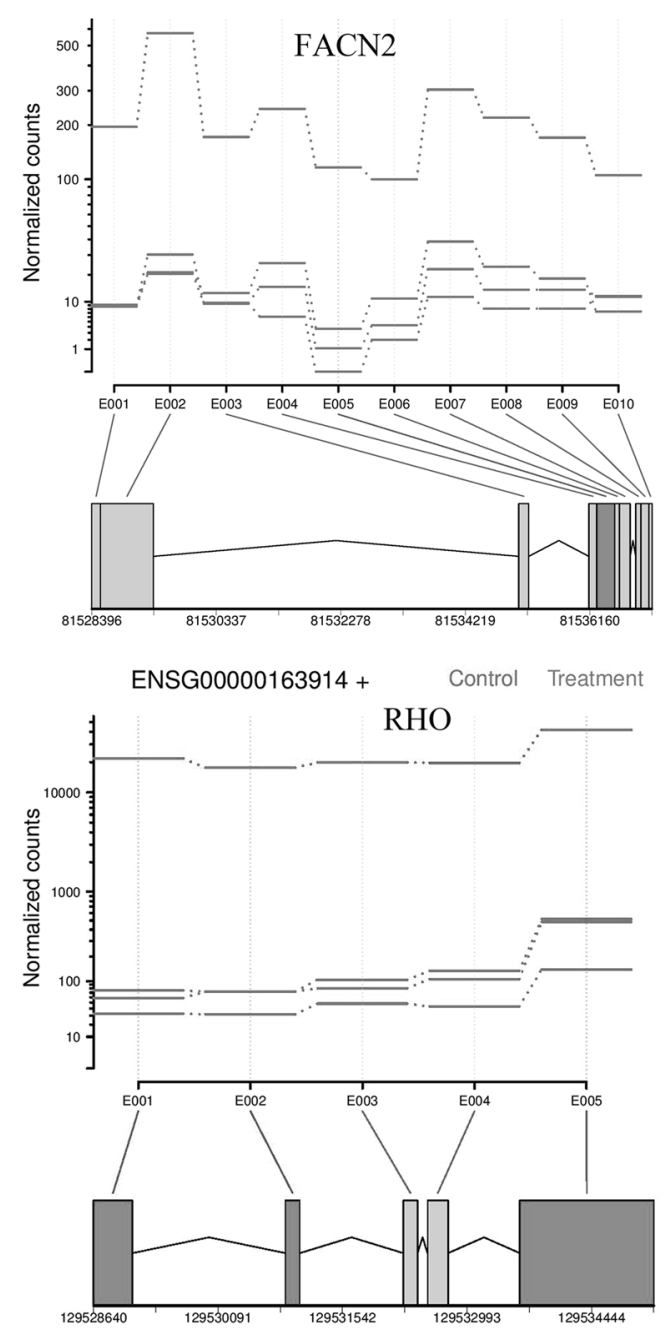

ENSG00000132915 - Control Treatment
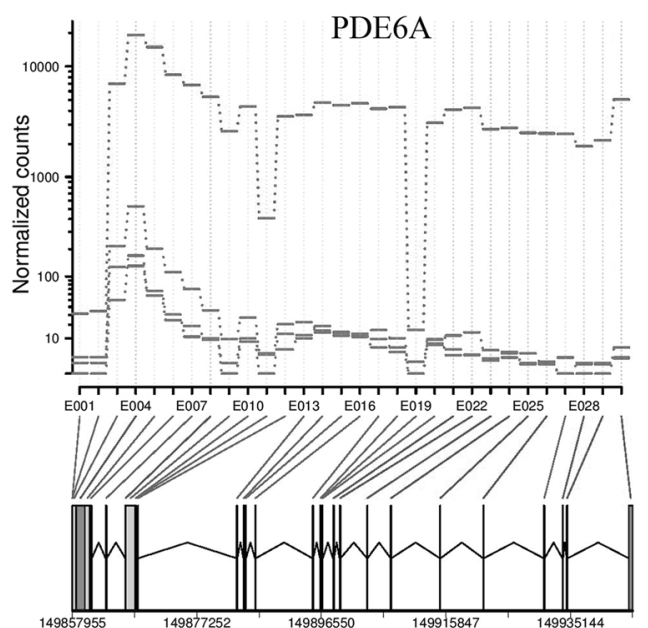

ENSG00000114349+ Control Treatment
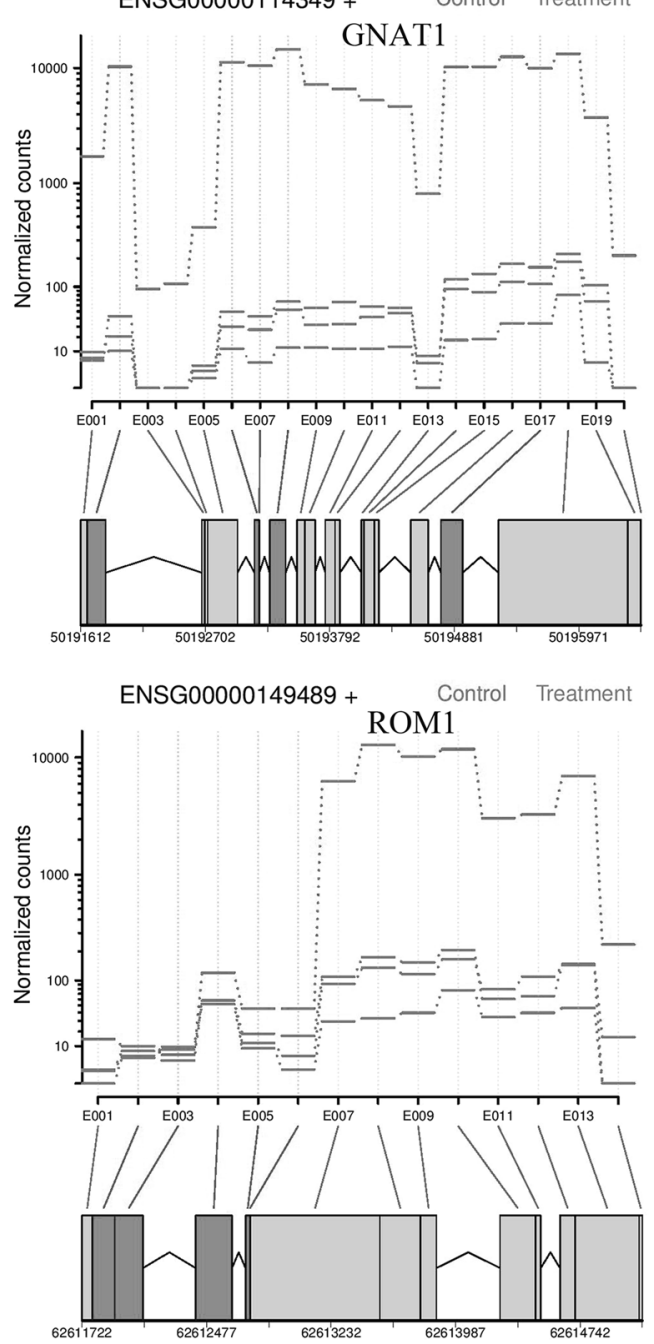

ENSG00000133256 + Control Treatment

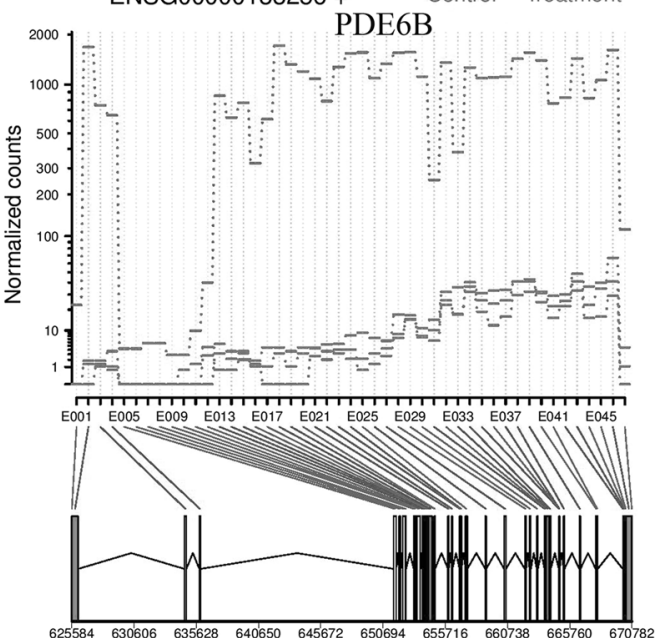

Fig. 2 (Continued) 
Table 2 Biological process classification between control and treated samples by PANTHER analysis

\begin{tabular}{lll}
\hline Biological process & $\begin{array}{l}\text { Fold } \\
\text { enrichment }\end{array}$ & $P$ value \\
\hline mRNA processing & 1.68 & $2.91 \mathrm{E}-02$ \\
Phototransduction & 3.91 & $1.28 \mathrm{E}-03$ \\
$\begin{array}{l}\text { Photoreceptor cell development } \\
\text { proteasome-mediated ubiquitin- } \\
\text { dependent protein catabolic process }\end{array}$ & 3.01 & $3.54 \mathrm{E}-04$ \\
$\begin{array}{l}\text { regulation of GTPase activity } \\
\text { negative regulation of transcription by }\end{array}$ & 1.84 & $3.70 \mathrm{E}-02$ \\
\begin{tabular}{l} 
RNA polymerase II \\
\hline
\end{tabular} & 1.68 & $1.96 \mathrm{E}-02$ \\
\end{tabular}

\section{Validation of intron retention in phototransduction genes}

To identify differences in splicing profiles, we performed large-scale transcriptome analyses between control and treated samples. To validate some of these findings, we performed RT-PCR experiments in treated and control samples in the key genes involved in phototransduction, in biological triplicates. The RT-PCR analysis showed intron retention in $R H O$, GNAT2, ROM1, FSCN2, and FSCN1 genes (Fig. 3). These data suggest that altered splicing in RP11 may be related to disruption of specific biological processes that cause the unique phenotypes observed in patients.

\section{Discussion}

The human PRPF31 protein is essential in the assembly and stability of U4/U6.U5 tri-snRNPs. Disease causing variants in this general splicing factor gene cause to RP11, retinal restricted disease, and the basis of this specificity is not yet clarified. Despite a lot of studies in immortalized cell lines and animal models, there are no reports for PRPF31-related RP11 that define the altered splicing of phototransduction genes in human retinal tissue. To obtain a more understanding of the RP11 pathomechanism, we used siRNA to reduce PRPF31 protein in HORCs and analyzed transcriptome-wide changes by RNA sequencing. Transcriptome analysis showed that the altered splicing via differential exon usage and intron retention is more related to pre-mRNA splicing program and phototransduction genes.

For analyzing the PRPF31 protein deficiency in the retina, we used DEU and intron retention analysis to study the alternative splicing in HORC. DEU produces different products of functional genes from a single genomic locus, which induces the diversity of gene products, encoded by the genome. In this study, amongst the 683,196 analyzed exons, 3170 exons showed DEU. For example, we observed DEUs in the RHO, ROM1, FSCN2, GNAT1, PDE6A, and
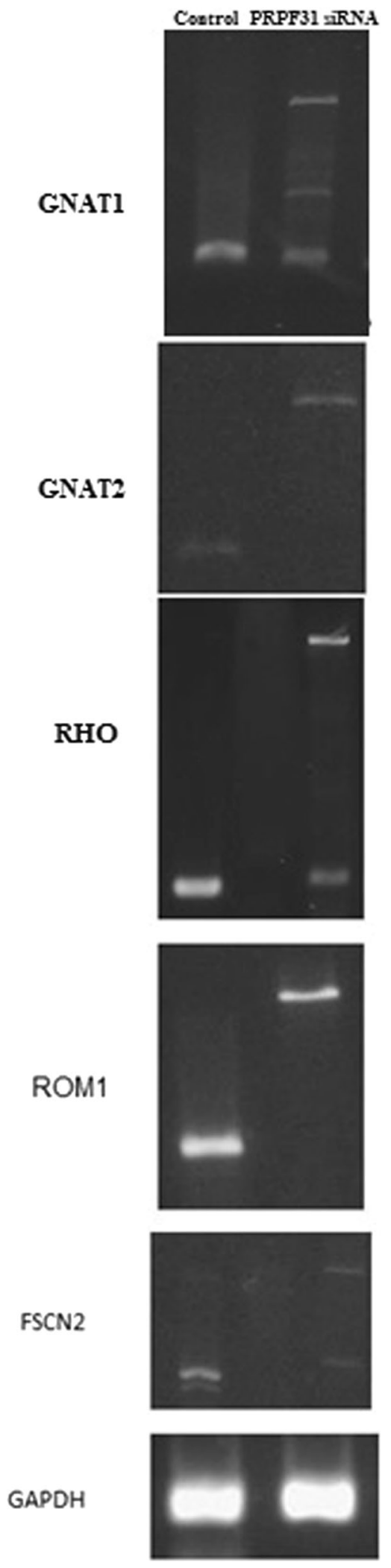

Fig. $3 \mathrm{Gel}$ electrophoresis of RT-PCR biological replication of differential alternative splicing events between control and treated samples. Gel electrophoresis showed intron retention in RHO, GNAT2, ROM1, GNAT1, and FSCN2 in PRPF31 reduced samples. Data are representative of at least three independent experiments 
$P D E 6 B$ genes, involved in phototransduction and other PRPFs families (PRPF3, PRPF4, PRPF8, and PRPF19) involved in pre-mRNA splicing. Also, several pathways, including RNA processing, regulation of GTPase activity, and photoreceptor cell development were deregulated in PRPF31 reduced samples. Indeed, we found that PRPF31 reduction causes intron retention in several phototransduction genes such as $R H O, R O M 1, F S C N 2$, and GNAT1.

One of the most affected altered splicing genes is rhodopsin. Rhodopsin forms over $90 \%$ of the proteins in the disc membranes of the rod photoreceptors and is necessary for low-light vision [27]. Proper production of the RHO is essential for the precise function of photoreceptors and any mis-splicing can cause to retinal degeneration [28]. Also, there was altered splicing of several components of PDE6 (phosphodiesterase 6) complex which controls intracellular cGMP levels by hydrolyzing cGMP in response to light activation and has an important role in the phototransduction [29]. Degeneration of photoreceptors is identified in a mouse model Pde6a variants. These variants alter the catalytic activity of PDE6A that is required for the preservation of $P D E 6 B$ levels in the retina [30]. Disease causing variants in the $P D E 6 B$ and $P D E 6 B$ cause autosomal recessive $\mathrm{RP}$ [31].

In addition, we observed altered splicing in GNAT1, FSCN2, and ROM1. GNAT1 has an essential role in phototransduction and encodes the $\alpha$ subunit of the rod transducin protein [32]. Mouse model gnat1 knockout showed a loss of rod photoreceptors function [33]. FSCN2 encodes a member of the fascin protein family members and has an important role in morphogenesis of photoreceptors outer segments [34]. $\mathrm{FSCN}_{2}{ }^{+/ \mathrm{p}}$ and $\mathrm{FSCN} 2^{+/ g}$ mice showed progressive degeneration of photoreceptors outer segment [35].

Also, our results showed the altered splicing of PRPFs genes suggest the capability of splicesome to maintain homeostasis of its distinct component.

To date, a few PRPF31 related RP11 studies have been reported from zebrafish [14] and $\operatorname{Prpf} 31^{+/-}$mice model [36]. Mice model of Prpf $31^{+/-}$did not display any degeneration in photoreceptors, but revealed alteration in retinal pigment epithelium. Microarray analysis of zebrafish model of Prpf31 knockdown showed altered splicing of retinal specific genes (gnat1, rx1, crx1, and rx3), consistent with the results of our HORC model. However, it should be considered that the PRPF31 deficiency leads to retinalspecific phenotype in human [37]. Therefore, there is an increasing demand for the characterization of the human transcriptome. Buskin et al., have investigated the cellular pathology and splicing program of RPE and retinal organoids derived from RP11 patients and observed that the PRPF31 deficiency disrupts the splicing program restricted to the retinal cells [38]. Also, Wickramasinghe et al., study has shown that the PRPF8 depletion in breast adenocarcinoma led to altered splicing of introns with weak 5 ' splice sites [39]. Disruption of the splicing program raises the questions relating to the functional effects of the altered splicing isoforms in the retina. Importantly, our transcriptomics analysis suggests that the retinal specific genes associated with the phototransduction are the most affected retinal genes in PRPF31-related RP11samples.

The retina is one of the fast metabolizing tissues and expresses the high volumes of spliceosomal snRNPs and processes the largest volume of pre-mRNA [7-9]. So, any alteration of splicing factors can be problematic for highly demanding photoreceptor cells.

Variants in PRPFs affect all splicing in the transcriptome, but retinal specific genes seem to be more susceptible than to the reduction of general splicing factors. However, retinal mis-spliced transcripts have not yet reported and more studies are necessary for comparing splicing in normal retina and patients. Also, photoreceptors are in postmitotic stage and any mis-spliced transcripts may collect in the tissue over time leading to increased cell death.

Our study recruited four samples and although this is a small number for performing comprehensive analysis, our results provided guidelines about the splicing program in the RP11 patients' retina. Also, our transcriptome analysis does not investigate the consequence of mis-spliced transcripts at the protein level and more analysis is required in the proteome level.

In conclusion, our findings confirm the role of PRPF31 for proper splicing of genes involved in phototransduction and its role in the core spliceosome that is required in regulating the pre-mRNA processing.

\section{Data availability}

The RNA-Seq data from this publication have been submitted to the NCBI (http://www.ncbi.nlm.nih.gov) under the accession numbers SRX5107787-SRX5107790.

Acknowledgements We thank Katarina Cisarova (Unit of Medical Genetics, University of Lausanne) for helpful comments on the paper and Dr Mozhgan Rezaei Kanavi and Dr Hamid Ahmadieh for providing posterior samples in the research.

\section{Compliance with ethical standards}

Conflict of interest The authors declare that they have no conflict of interest.

Publisher's note Springer Nature remains neutral with regard to jurisdictional claims in published maps and institutional affiliations.

\section{References}

1. Corton M, Ávila-Fernández A, Campello L, Sánchez M, Benavides B, López-Molina MI, et al. Identification of the photoreceptor 
transcriptional co-repressor SAMD11 as novel cause of autosomal recessive retinitis pigmentosa. Sci Rep. 2016;6:35370.

2. Dias MF, et al. Molecular genetics and emerging therapies for retinitis pigmentosa: basic research and clinical perspectives. Prog Retin Eye Res. 2018;63:107-31.

3. Van Cauwenbergh C, Coppieters F, Roels D, De Jaegere S, Flipts $\mathrm{H}$, De Zaeytijd J, et al. Mutations in splicing factor genes are a major cause of autosomal dominant retinitis pigmentosa in Belgian families. PLoS ONE. 2017;12:e0170038.

4. Růžičková Š, Staněk D. Mutations in spliceosomal proteins and retina degeneration. RNA Biol. 2017;14:544-52.

5. Carey KT, Wickramasinghe VO. Regulatory potential of the RNA processing machinery: implications for human disease. Trends Genet. 2018;34:279-90.

6. Lee Y, Rio DC. Mechanisms and regulation of alternative premRNA splicing. Annu Rev Biochem. 2015;84:291-323.

7. Liu MM, Zack DJ. Alternative splicing and retinal degeneration. Clin Genet. 2013;84:142-9.

8. Pinelli M, Carissimo A, Cutillo L, Lai C-H, Mutarelli M, Moretti $\mathrm{MN}$, et al. An atlas of gene expression and gene co-regulation in the human retina. Nucleic Acids Res. 2016;44:5773-84.

9. Tanackovic G, Ransijn A, Thibault P, Abou Elela S, Klinck R, Berson EL, et al. PRPF mutations are associated with generalized defects in spliceosome formation and pre-mRNA splicing in patients with retinitis pigmentosa. Hum Mol Genet. 2011;20:2116-30.

10. Padgett RA. New connections between splicing and human disease. Trends Genet. 2012;28:147-54.

11. Mordes D, Luo X, Kar A, Kuo D, Xu L, Fushimi K, et al. PremRNA splicing and retinitis pigmentosa. Mol Vis. 2006;12:1259.

12. Tanackovic G, Ransijn A, Thibault P, Elela SA, Klinck R, Berson EL, et al. Generalized defects in spliceosome composition and pre-mRNA splicing are associated with retinitis pigmentosa in humans. Investig Ophthalmol Vis Sci. 2011;52:1639.

13. Bujakowska K, Maubaret C, Chakarova CF, Tanimoto N, Beck SC, Fahl E, et al. Study of gene-targeted mouse models of splicing factor gene Prpf31 implicated in human autosomal dominant retinitis pigmentosa (RP). Investig Ophthalmol Vis Sci. 2009;50:5927-33.

14. Linder B, Dill H, Hirmer A, Brocher J, Lee GP, Mathavan S, et al. Systemic splicing factor deficiency causes tissue-specific defects: a zebrafish model for retinitis pigmentosa. Hum Mol Genet. 2010;20:368-77.

15. Graziotto JJ, Farkas MH, Bujakowska K, Deramaudt BM, Zhang Q, Nandrot EF, et al. Three gene-targeted mouse models of RNA splicing factor RP show late-onset RPE and retinal degeneration. Investig Ophthalmol Vis Sci. 2011;52:190-8.

16. Schaffert N, Hossbach M, Heintzmann R, Achsel T, Lührmann R. RNAi knockdown of hPrp31 leads to an accumulation of U4/U6 di-snRNPs in Cajal bodies. EMBO J. 2004;23:3000-9.

17. Rivolta C, McGee TL, Frio TR, Jensen RV, Berson EL, Dryja TP. Variation in retinitis pigmentosa-11 (PRPF31 or RP11) gene expression between symptomatic and asymptomatic patients with dominant RP11 mutations. Hum Mutat. 2006;27:644-53.

18. Cao H, Wu J, Lam S, Duan R, Newnham C, Molday RS, et al. Temporal and tissue specific regulation of RP-associated splicing factor genes PRPF3, PRPF31 and PRPC8-implications in the pathogenesis of RP. PLoS ONE. 2011;6:e15860.

19. Abu-Safieh L, Vithana EN, Mantel I, Holder GE, Pelosini L, Bird $\mathrm{AC}$, et al. A large deletion in the adRP gene PRPF31: evidence that haploinsufficiency is the cause of disease. Mol Vis. 2006;12:384-8.

20. Frio TR, Wade NM, Ransijn A, Berson EL, Beckmann JS, Rivolta C. Premature termination codons in PRPF31 cause retinitis pigmentosa via haploinsufficiency due to nonsense-mediated mRNA decay. J Clin Investig. 2008;118:1519-31.
21. Azizzadeh Pormehr L, Daftarian N, Ahmadian S, Rezaei Kanavi M, Ahmadieh H, Shafiezadeh M. Human organotypic retinal flatmount culture (HORFC) as a model for retinitis pigmentosa11. J Cell Biochem. 2018;119:6775-83.

22. Jiang M, Chen G. High Ca 2+-phosphate transfection efficiency in low-density neuronal cultures. Nat Protoc. 2006;1:695.

23. Kim D, Langmead B, Salzberg SL. HISAT: a fast spliced aligner with low memory requirements. Nat Methods. 2015;12:357.

24. Anders S, Reyes A, Huber W. Detecting differential usage of exons from RNA-seq data. Genome Res. 2012. https://doi.org/10. 1101/gr.133744.111.

25. Trapnell C, Roberts A, Goff L, Pertea G, Kim D, Kelley DR, et al. Differential gene and transcript expression analysis of RNA-seq experiments with TopHat and Cufflinks. Nat Protoc. 2012;7:562.

26. Bullard JH, Purdom E, Hansen KD, Dudoit S. Evaluation of statistical methods for normalization and differential expression in mRNA-Seq experiments. BMC Bioinform. 2010;11:94.

27. Filipek S, Stenkamp RE, Teller DC, Palczewski K. G proteincoupled receptor rhodopsin: a prospectus. Annu Rev Physiol. 2003;65:851-79.

28. Palczewski K. G protein-coupled receptor rhodopsin. Annu Rev Biochem. 2006;75:743-67.

29. Qureshi BM, Behrmann E, Schöneberg J, Loerke J, Bürger J, Mielke T, et al. It takes two transducins to activate the cGMPphosphodiesterase 6 in retinal rods. Open Biol. 2018;8:180075.

30. Wang T, Reingruber J, Woodruff ML, Majumder A, Camarena A, Artemyev NO, et al. The PDE6 mutation in the rd10 retinal degeneration mouse model causes protein mislocalization and instability and promotes cell death through increased ion influx. J Biol Chem. 2018;293:15332-46.

31. Cheng L-L, Han R-Y, Yang F-Y, Yu X-P, Xu J-L, Min Q-J, et al. Novel mutations in PDE6B causing human retinitis pigmentosa. Int J Ophthalmol. 2016;9:1094.

32. Carrigan M, Duignan E, Humphries P, Palfi A, Kenna PF, Farrar GJ. A novel homozygous truncating GNAT1 mutation implicated in retinal degeneration. Br J Ophthalmol. 2015. https://doi.org/10. 1136/bjophthalmol-2015-306939.

33. Calvert P, Krasnoperova N, Lyubarsky A, Isayama T, Nicolo M, Kosaras B, et al. Phototransduction in transgenic mice after targeted deletion of the rod transducin $\alpha$-subunit. Proc Natl Acad Sci USA. 2000;97:13913-8.

34. Wada Y, Abe T, Takeshita T, Sato H, Yanashima K, Tamai M. Mutation of human retinal fascin gene (FSCN2) causes autosomal dominant retinitis pigmentosa. Investig Ophthalmol Vis Sci. 2001;42:2395-400.

35. Yokokura S, Wada Y, Nakai S, Sato H, Yao R, Yamanaka H, et al. Targeted disruption of FSCN2 gene induces retinopathy in mice. Investig Ophthalmol Vis Sci. 2005;46:2905-15.

36. Farkas MH, Lew DS, Sousa ME, Bujakowska K, Chatagnon J, Bhattacharya SS, et al. Mutations in pre-mRNA processing factors 3,8 , and 31 cause dysfunction of the retinal pigment epithelium. Am J Pathol. 2014;184:2641-52.

37. Matlin AJ, Clark F, Smith CW. Understanding alternative splicing: towards a cellular code. Nat Rev Mol Cell Biol. 2005;6:386.

38. Buskin A, Zhu L, Chichagova V, Basu B, Mozaffari-Jovin S, Dolan D, et al. Disrupted alternative splicing for genes implicated in splicing and ciliogenesis causes PRPF31 retinitis pigmentosa. Nat Commun. 2018;9:4234.

39. Wickramasinghe VO, Gonzàlez-Porta M, Perera D, Bartolozzi AR, Sibley CR, Hallegger M, et al. Regulation of constitutive and alternative mRNA splicing across the human transcriptome by PRPF8 is determined by $5^{\prime}$ splice site strength. Genome Biol 2015;16:201. 